\title{
PID Controller Tuning using ACO Algorithm for AVR Systems
}

\author{
Harsha S Anantwar, Anirudh Suresh Ramachandran
}

\begin{abstract}
This paper features an effective technique to device the parameters of PID controllers for utilization together with an Automatic Voltage Regulator System (AVR). The quintessential goal is to acquire a good load disturbance response by minimizing the performance index/(Integral time square error). Simultaneously, the transient response is assured by limiting maximum overshoot, settling time and rise time of the step response to minimal values. For achieving these goals, optimum and quick tuning of the parameters $(\mathrm{Kp}, \mathrm{Ki}$, and $\mathrm{Kd})$ is essential. In an effort to accomplish the aforementioned, the paper put forth an algorithm developed based on the Ant Colony Optimization technique (ACO) to decide optimal gains of PID controller and for getting optimal performance within an AVR system. Simulation results establish superior control response may be accomplished in comparison with methods like conventional tuning method (trial and error) and built-in genetic algorithm (GA) took-kit.e.
\end{abstract}

\section{INTRODUCTION}

\section{$\mathrm{P}$} roportional-integral-derivative controllers are typically employed to regulate and stabilize the time domain behavior of dynamic plants and systems. These controllers are widely accepted owing to their simple structure, good performance characteristics for a broad range of conditions and environments Despite the straightforward structure of this controller there is necessity is to tune its parameters; proportional gain (Kp), integral gain (Ki), and derivative time constant gain (Kd) effectively and suitably [1]. Conventionally, the problem has been handled by a trial and error method or by using older algorithms such as the Ziegler-Nichols method. However, it is often problematic and tiresome to obtain optimal PID parameters with these methods. In order to reduce the complication compared to traditional method of tuning the PID controller parameters, many optimization methods have already been developed, like, fuzzy logic [2]-[3], self-tuning methods [4]-[5],particle swarm optimizations[6],artificial bee optimization[7], and pattern recognition [8] genetic algorithm (GA) [9], particle swarm optimization [10]. These techniques tend to be quite effective during their tuning of the PID controller. Better methods not only enable a quicker tuning, in addition, provide us with better performance and enable for

\section{Revised Manuscript Received on February 05, 2020.}

* Correspondence Author

Harsha S Anantwar*, Electrical and Electrical Engineering department, Dayananda Sagar College of Engineering , Bangalore. Email: hanantwar@yahoo.com

Anirudh Suresh Ramachandran, Electrical and Electrical Engineering department, Dayananda Sagar College of Engineering , Bangalore.

(C) The Authors. Published by Blue Eyes Intelligence Engineering and Sciences Publication (BEIESP). This is an open access article under the CC BY-NC-ND license (http://creativecommons.org/licenses/by-nc-nd/4.0/)
Keywords : ACO, AVR and PID

widespread utilization of the controller Within this research, we have developed the challenge of design PID controllers , for usage within the AVR system by applying the ant colony optimization algorithm (ACO) [11].The algorithm works on the closed feedback loop that is formed by the AVR and the PID controller. Researchers have confirmed the promising potential of ACO to competently explore and locate an optimal solution. The procedure followed influenced by examining the proven fact that ants can discover the shortest path between their nest and as well as a food source [12]

ACO is basically a metaheuristic approach suited for finding solutions of complex optimization problems. Some of the examples include the traveling salesman problem, quadratic assignment problem, vehicle routing, scheduling [13]. Natural ants inspire a lot of the behavior used in this algorithm .some of main traits are listed here: (1) artificial ants prevail in colonies of cooperating individuals, (2) communicates indirectly by the dumping chemical pheromone. Simililary in ACO ,a colony of artificial ants work together to search and explore solutions in given domain in order, to find the optimal solution

\section{AVR SYSTEM}

AVR comprises of four main components the amplifier, exciter, sensor.PID controller is added so as to help control the AVR which in turn regulates the reactive power and voltage magnitude. An AVR system mainly regulates the fluctuations in voltage, these mainly happen because of the variation within the load. This variation might lead to severe impairment of the system and equipment. To prevent the variation in the voltage and mitigate damage to equipment, AVR is provided in more than one point in the system. Now, the generator voltage in the AVR is sensed by direct connection to the terminals of the generator. This sensed voltage is then fed to a measurement unit which converts it into a low-value direct voltage proportional to the generator voltage. Further, the terminal voltage of a generator obtained through a potential transformer which is then rectified, filtered and compared with a reference voltage. Thus, the AVR system detects the difference between these two and generate an error voltage. The AVR can be tuned effectively by making use of a PID controller, which in turn is optimally tuned by ACO algorithm. Controller output gives an appropriate signal to the main exciter, then the exciter adjusts the field current of the machine, to bring system voltage nearer to the reference value. This helps to reduce the overvoltages which occur due to the sudden loss of load on the system or any other types of fluctuations which may occur due to variations in the load [14].The AVR system components are modeled using transfer functions, which considers only the time constant ignoring the saturation or other nonlinearities. These components can be in Fig.1 [15].

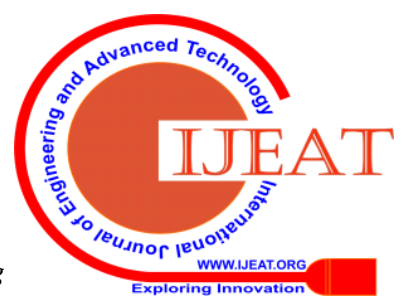




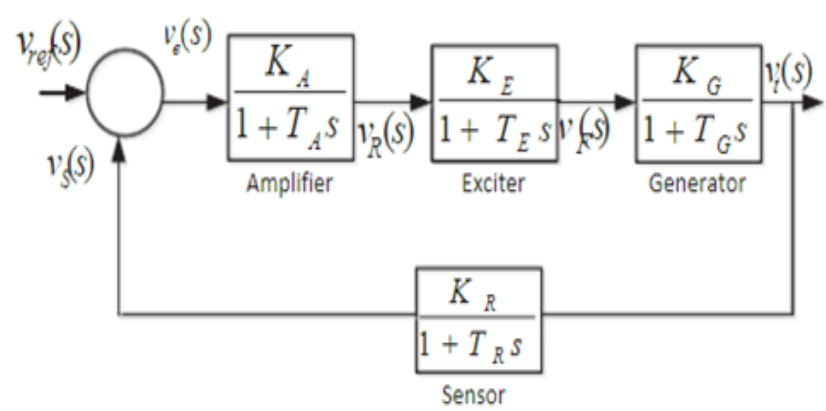

Fig.1-Simplified block diagram of an AVR system

\section{OPTIMIZATION PROBLEM}

An optimization problem is defined as selecting optimal values of PID controller parameter to enhance the response of the AVR system under varying load condition. In this work $\mathrm{Kp}, \mathrm{Ki}$ and $\mathrm{Kd}$ gains controller are optimally tuned using the ant colony algorithm. The transient state of system is important since the system must reach steady-state quickly and the time response of the system must be kept within the endorsed limits. [1,16] ] Hence, three key performance evalution criterias are utilized to characterize the performance systems ,these include: ITSE(integral time square error ), overshoot, and settling time.

\section{ANT COLONY OPTIMIZATION}

ACO is an evolutionary meta-heuristic algorithm. It is a graphical method of solving the problem and has been proven successful and effective to handle various hard combinatorial optimization problems. Main insight of ACO is to create a graphical model of the problem and search for the path the shortest path and best the cost. Artificial ants' traverse across the various nodes. A single ant has a very straightforward behavior and would be unable to find good paths or solutions on their own but with the help of cooperation among a number of them, they are able to find out the best path and hence the best solution. This cooperation happens because of deposition involving a chemical substance known as pheromone.

As the best way to find the solution is to test all the possibilities one by one, the ants as they roam through the paths deposit this chemical substance which the other ants can follow. Firstly, the way the ants choose a certain path is dependent on Pseudo-Random-Proportional Action Choice Rule which gives us the probability of which node the ants might choose the next step. An ant will move from node $x$ to node y with probability .

$$
P_{i j}=\frac{\left[\tau_{x y}\right]^{\alpha}\left[\eta_{x y}\right]^{\beta}}{\left[\tau_{x y}\right]^{\alpha}\left[\eta_{x y}\right]^{\beta}}
$$

Where ' $\alpha$ ' represents the relative importance of the pheromone trace in the problem and ' $\beta$ ' represents heuristic factor. The Eq.(1), the probability equation is a requital between the heuristi factor and the pheromone factor. Two types of different process that occur in the following algorithm is first because of the pheromone factor if there is a lot of ants taking a similar path then this then creates an autocatalytic process whereas due to the heuristic factor closer nodes are chosen with higher probability thus creating a greedy constructive heuristic process. Thus, Ants allocate the next elections in accordance with possibility equation. A round orientation is finished considering that all the nodes within the problem are visited and traveled. After this , amount of pheromone trace is updated in accordance with Eq. (2) and Eq.(3) given below. In other words, pheromone updation is intended to allocate a larger quantity of pheromone to paths with a lower cost (small tours). This value is assessed when the ant has completed a tour which consists of a cycle of' ' $n$ ' iterations . Then, it is used to update the amount of substance beforehand put down on the path, based on the subsequent rules

$\operatorname{txy}(\mathrm{t}+\mathrm{a})=(1-\mathrm{E}) \tau x y+\Delta \operatorname{txy}$

$\Delta \operatorname{txy}(\mathrm{t})=\sum_{\mathrm{k}=1}^{\mathrm{a}} \Delta \mathrm{txy}$

where a is number of ants . $E$ is the persistence of the trail in the generations. The pheromone-updating rule was focusing on trying to simulate the transition in the total sum of pheromone stemming from both the addition of latest pheromone deposited by ants upon visiting the edges plus some lost as a consequence of pheromone evaporation. The algorithm terminates iterating either when an ant finds a solution or whenever the maximum number of generations has been executed. $[17,18]$

\section{TUNNING OF PID CONTROLLER USING ACO ALGORITHM}

The PID controller is developed using ACO to the optimally control AVR system can be seen in Fig.2

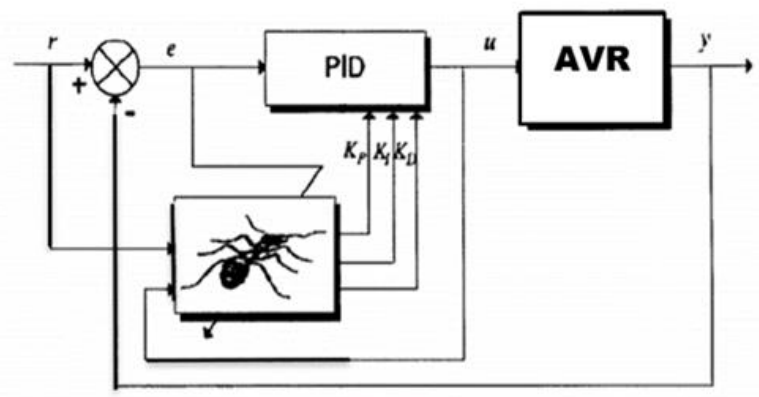

Fig2- ACO optimized of PID controller

The optimal design of PID controller means to select the best parameters $\mathrm{Kp}, \mathrm{Ki}$ and $\mathrm{Kd}$ such that the performance indexes of the step response of the system are kept minimal. . To develop graph representation involving an optimal problem, the vectors are considered as paths between the nests. All the paths are considered as nodes .Start and end node are also considered. Within the tour,

the ant must visit all of the nodes by selecting a pathway from the start node to the end node. The purpose of ACO would be to search for the best tour having the lowest value of the cost function or objective function amongst all the three paths. All of the of ants deposit pheromone onset of each new path. Subsequently the pheromones are updated in pheromone updating rule $[17,18]$.

Now the pseudo-code of the algorithm for use in PID -AVR system is presented below :

The ACO pseudo-code for the optimal design of PID is as follows

Set parameters and initialize pheromone trail

Start iteration from node 1 to 1000

Determine the probability distribution of the nodes 
Determine the probability sum

The cost function is calculated

Best cost is determined

Pheromone trails updated

End iteration

Display parameters with the best cost

Larger the nodes gives more precise results. Trails are updated only after having constructed complete path and the solution . Each path represents the performance indexes (cost function) on the load disturbance response and transient response for a set of parameters (Kp, Ki\& Kd). The). The ACO parameters used are: $\alpha=0.8, \beta=0.2$, Evaporation Rate $=0.2$, nodes $=1000$, number of ants $=10$, number if iterations $=10$. In this paper as stated the number nodes used are 1000, a greater number of nodes produced better accuracy, though the solution is found much slower. Fig.3 represents the graphical movement by which the ant colony algorithm works. Each column represents the PID parameters, while each graphical node represents a numerical solution for corresponding parameters (Kp, Ki, and $\mathrm{Kd}$ ). Ants as stated move about these nodes trying to discover the best solution. Trails are updated only after creating complete path and the solution found.

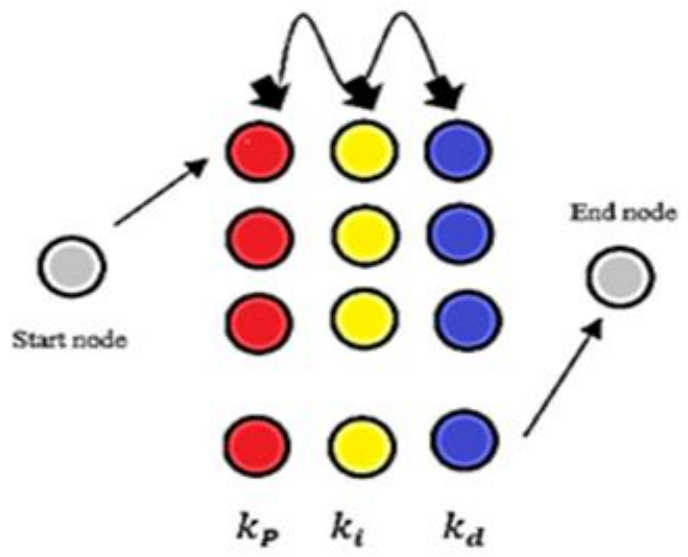

Fig3-Ant movement

\section{SIMULATION RESULTS}

The simulation results of ACO based optimal tuned AVR is presented in this section. To implement the ACO algorithm we design a Simulink model of AVR [1] is presented in Fig.4

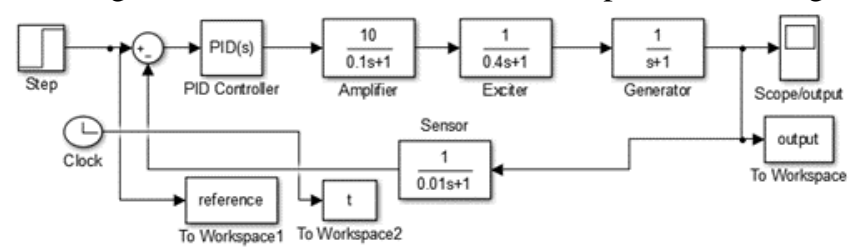

Fig4- Simulink model

The ACO algorithm is tested for various performance indices (objective function) such as ITSE, IAE, ISE and ITAE employed for solving an optimization problem (optimal tuning of controller parameter) is tabulated in Table 1. From TableI, it is seen that the objective function is consistently minimum in case of the ITSE compared to all the other performance indexes.

TABLE I: Performance index tabulation

\begin{tabular}{|l|l|l|c|c|}
\hline S/NO & ITSE & IAE & ISE & ITAE \\
\hline 1 & $\mathbf{1 0 . 6 1}$ & 269.2051 & 109.10 & 251.82 \\
\hline 2 & $\mathbf{1 0 . 2 7}$ & 231.1285 & 103.67 & 231.16 \\
\hline 3 & $\mathbf{1 0 . 9 1}$ & 274.6025 & 106.12 & 237.22 \\
\hline
\end{tabular}

\begin{tabular}{|l|l|l|l|l|}
\hline 4 & $\mathbf{1 1 . 6 4}$ & 220.2894 & 104.38 & 232.32 \\
\hline 5 & $\mathbf{1 1 . 5 6}$ & 223.5646 & 105.12 & 251.32 \\
\hline 6 & $\mathbf{1 2 . 4 6}$ & 214.9676 & 105.16 & 218.13 \\
\hline 7 & $\mathbf{1 2 . 8 0}$ & 215.8716 & 109.96 & 220.60 \\
\hline 8 & $\mathbf{1 1 . 9 6}$ & 224.4941 & 105.14 & 215.12 \\
\hline 9 & $\mathbf{1 1 . 4 3}$ & 216.0378 & 108.80 & 220.40 \\
\hline 10 & $\mathbf{1 0 . 3 6}$ & 230.6225 & 107.23 & 230.12 \\
\hline
\end{tabular}

Comparison of AVR response for various performance indices is illustrated in Fig.5, From that, we can notice that the ITSE gives us the best response characterizes giving the fastest settling time and overshoot

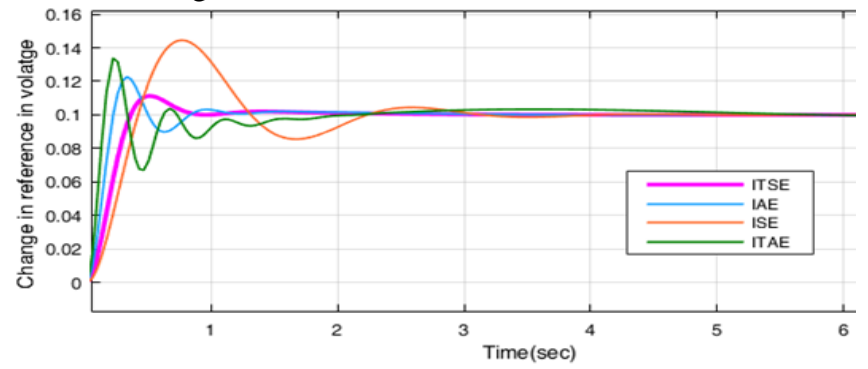

Fig5- Comparison of AVR response for various performance indices for $1 \%(0.1$ p.u 0 step disturbance condition

The results obtained through the ACO algorithm and two other methods for validating the effectiveness of ACO. The results obtained by using the GA algorithm and a conventional method (trial and error) are presented in Fig. 6

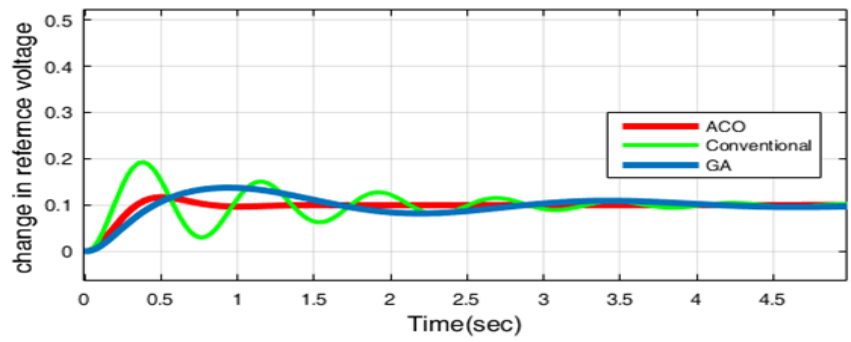

Fig6- Comparison of AVR response with ACO ,GA and Conventional method optimal tuned PID controller for $1 \%$ step disturbance condition. citation [8].

Table II presented controller parameters obtained by the three above mentioned methods for $1 \%$ step change in reference voltage change to the AVR system.

Table III summarizes time domain specification of AVR response for $1 \%$ change in reference voltage for GA, ACO and Conventional method

TABLE II : Optimal PID Controller parameters

\begin{tabular}{|l|l|l|c|}
\hline Specification & \multicolumn{1}{|c|}{ GA } & \multicolumn{1}{|c|}{ Conventional } & ACO \\
\hline $\mathrm{Kp}$ & 2.7 & 0.4661 & $\mathbf{0 . 8 8 4 0 2}$ \\
\hline $\mathrm{Ki}$ & 8 & 1.4671 & $\mathbf{0 . 5 6 3 5 8}$ \\
\hline $\mathrm{Kd}$ & 0.34 & 0.1490 & $\mathbf{0 . 2 1 9 1 3}$ \\
\hline
\end{tabular}

TABLE III: Time domain specification of AVR response

\begin{tabular}{|c|c|c|c|}
\hline Specification & Conventional & GA & ACO \\
\hline $\begin{array}{l}\text { Settling Time } \\
\text { (Sec) }\end{array}$ & 5 & 4 & 3.5 \\
\hline ITSE & 186.65 & 97.98 & 0.10466 \\
\hline
\end{tabular}

Published By:

Blue Eyes Intelligence Engineering 562 \& Sciences Publication 


\begin{tabular}{l|l|l|l|}
\hline Overshoot & $220 \%$ & $38.1 \%$ & $\mathbf{2 5 . 9 4 \%}$ \\
\hline paper.
\end{tabular}

\section{CONCLUSION}

In this work, an improved concept to design and model the PID controller for usage in an AVR system in order to optimize and enhance its response is featured. The optimization of the PID controller is made by using three key performance indexes namely maximum overshoot, settling time, and ITSE. It is realized that for the particular usage the ITSE proves to be a better fit compared to IAE, ISE, and ITAE. The results obtained by using genetic algorithm(GA) and conventional method are also featured for comparison and contrast. It can be concluded that the proposed method, the ant colony algorithm provides a better response on the three Performance indexes compared to the other methods. The characteristic feature of the presented algorithm unique include that it can be (i) it can be implemented relatively easy, (ii) flexible in nature and hence upon changing a few parameters allows for use in different applications (iii) search in parallel provides a guaranteed and definitive converge.

\section{REFERENCES}

1. Onsivilai and P. Pao-La-Or, "Application of adaptive tabu search for optimum PID controller tuning AVR system," WSEAS Transactions on Power Systems, vol. 3, no. 6, pp. 495- 506, 2008.

2. S. Tzafestas and N. P. Papanikolopoulos, "Incremental fuzzy expert PID control," IEEE Transactions on Industrial Electronics, vol. 37, no. 5, pp. 365-371, 1990.

3. Visioli, "Tuning of PID controllers with fuzzy logic," IEE Proceedings: Control Theory and Applications, vol. 148, no. 1, pp. 1-8, 2001.

4. Comparison between optimally-tuned PID with self-tuning PID for steam temperature regulation Mazidah Tajjudin ; Mohd Hezri Fazalul Rahiman ; Norlela Ishak ; Ramli Adnan ; Hashimah Ismail Year: 2012 | Volume: 2 | Conference Paper | Publisher: IEEE

5. Self-tuning PID control structures P.J. Gawthrop IEE Colloquium on Getting the Best Our of PID in Machine Control Year: 1996 | Conference Paper | Publisher: IET

6. Optimal PID controller design for AVR system using particle swarm optimization algorithm MohammadSadegh Rahimian Kaamran Raahemifar 2011 24th Canadian Conference on Electrical and Computer Engineering(CCECE) Year: 2011 | Conference Paper | Publisher: IEEE

7. Optimum Design of Fractional Order PID Controller for an AVR System Using an Improved Artificial Bee Colony ZHANG Dong-Li TANG Ying-Gan ,Acta Automatica Sinica Volume 40, Issue 5, May 2014, Pages 973-979

8. G. Zhou and J. D. Birdwell, "Fuzzy logic-based PID autotuner design using simulated annealing," in Proceedings of the IEEE/IFAC Joint Symposium on Computer-Aided, pp. 67-72, 1994.

9. P. Wang and D. P. Kwok, "Optimal design of PID process controllers based on genetic algorithms," Control Engineering Practice, vol. 2, no. 4, pp. 641-648, 1994.

10. P. Wang and D. P. Kwok, "Optimal design of PID process controllers based on genetic algorithms," Control Engineering Practice, vol. 2, no. 4, pp. 641-648, 1994.

11. Y. Mitsukura, T. Yamamoto, and M. Kaneda, "A design of self turning PID controllers 'using a genetic algorithm," in Proceedings of the American Control Conference, pp. 1361- 1365, San Diego, Calif, USA, 1999.

12. S. E. Selvan, S. Subramanian, and S. T. Solomon, "Novel technique for PID tuning by particle swarm optimization," in Proceedings of the 7th Annual Swarm Users/Researchers Conference (Swarm Fest '03), 2003.

13. Y. T. Hsiao, C. L. Chuang, and C. C. Chien, "Ant colony optimization for designing of PID controllers," in Proceedings of the IEEE international Symposium on Computer Aided Control Systems Design, Taipei, Taiwan, 2004.

14. S.J. Huang, "Enhancement of hydroelectric generation scheduling using ant colony system-based optimization approaches," IEEE Transactions on Energy Conversion, vol. 16, no. 3, pp. 296-301, 2001.
15. J. Faiz "Analysis and Simulation of the AVR System and Parameters Variation Effects", School of Electrical and Computer Engineering, University of Tehran, Tehran, Iran 2007 International Conference on Power Engineering, Energy and Electrical Drives

16. for Designing of PID Controllers" 2004 IEEE International Symposium on Computer Aided Control Systems Design Taipei, Taiwan, September 24,2004

17. Ying-Tung Hsiao, Cheng-Long Chuang "Ant Colony Optimization Ibtissem Chiha, Noureddine Liouane, and Pierre Borne "Tuning PID Controller Using Multiobjective Ant Colony Optimization" Applied Computational Intelligence and Soft Computing, Volume 2012, Article ID 536326, 7 pages, Hindawi publication

18. Vivek Kumar Bhatt1, Dr. Sandeep Bhongade "Design of PID Controller in Automatic Voltage Regulator (AVR) System Using PSO Technique" International Journal of Engineering Research and Applications (IJERA)

\section{AUTHORS PROFILE}

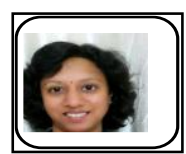

Harsha Anantwar did her Ph.D from Visvesvaraya Technological University, Karnataka. . She has published several research papers in reputed international journals and conferences. Her research area includes Voltage control and reactive power Management in off-grid power system and Smart Grid, . She is member of ISTE and IEEE.

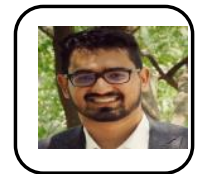

Anirudh Suresh Ramchandran did his Electrical engineering from Dayananda Sagar college of Engineering Bangalore , . He is currently pursuing his aters in electrical and computer engineering from UC Davis, California . His research interests include smart grid and operations research 